\title{
1 Oil transport in shale nanopores and micro-fractures: modeling
}

2

3

4

\section{and analysis}

Jiangfeng Cui ${ }^{\text {a* }}$

a Physical Science and Engineering Division, King Abdullah University of Science and Technology, Jeddah, Saudi Arabia

*Corresponding author. E-mail address: cuijiangfeng_pascal@126.com

\section{Abstract}

The mathematical modelling and analysis for the apparent permeability of shale oil with complicated compositions in dual-wettability shales (superior to existing single-component or single-wettability analysis), which is the core of this work, will help understand the detailed contributions of the considered mechanisms. Based on a previously established flow enhancement model, the effects of the mixed wettability and those triggered by asphaltenes, namely, the wettability alteration, the pore radius reduction, and the viscosity increment, are taken into account by respective corrections of the variables in the model. The effect of the mixed wettability is quantified by the water-wet area ratio, while the surface coverage, the adsorption thickness, and the volume fraction in the free phase are utilized to quantify the effects of asphaltenes. The volume fraction of asphaltenes in the free phase is related to that in both phases by the volume conservation equation. The full mathematical model and its several simplifications are achieved. Comprehensive sensitivity analysis and comparisons are carried out to demonstrate the effects of each variable (i.e., the pore radius, the initial water-wet surface ratio, the relative adsorption thickness, and the surface coverage) on: (1) the flow enhancement; (2) the relative importance of all mechanisms; (3) the differences among organic pores/inorganic pores/micro-fractures; and (4) 
the overall contribution of asphaltenes. The effect of different adsorption patterns (characterized by the relative adsorption thickness and the surface coverage) is also discussed.

\section{Keywords}

Shale oil, asphaltenes, mixed wettability, adsorption, mathematical model

\section{Introduction}

With the declining production of conventional resources, recently worldwide researchers switched their enthusiasm to enhanced recovery methods of unconventional resources, shale oil reserves for instance. Shale formations are rich in reserve, and even a slight increase in the recovery means a significant increment in the production. However, due to the ultra-low permeability and the poorly connected porosity of shales, the not well-known oil flow mechanisms in shales need more investigation (Bousige et al., 2016; Falk et al., 2015). Shales are featured by the organic matter and nanoscale pores, and some studies have shown the enhancement effect in organic nanopores. On the other hand, different from shale gas, crude oil has complicated compositions (e.g., resins and asphaltenes), and some previous works have targeted at the consequent formation damage. These characteristics should be both considered for a comprehensive research on the oil flow regularities in shale rocks, as such combination will induce some new scientific findings.

Primary MDS (Molecular dynamics simulation) researches by Wang et al. show the static adsorption (Wang et al., 2015b, 2015a) and dynamic flow (Wang et al., 2016a, 2016b) characteristics of shale oil. The concept of the flow enhancement (Strogatz et al., 2005) stemming from the boundary slip (Botan et al., 2011; Ho et al., 2011; Li et al., 2010; Neto et al., 2005; Thomas and McGaughey, 2009; Wang et al., 2010) is used to characterize the deviation from classic fluid dynamics. Results illustrate the distinct flow properties between organic and inorganic nanopores: oil flow in organic pores is much faster. In reality, MDS might not quantitatively describe the interfacial dynamics well (Secchi et al., 2016). Cui et al. (Cui et al., 2017) formulated and analyzed the liquid permeability of nanopores following Mattia's model (Mattia et al., 2015; Mattia and Calabro, 2012; Ritos et al., 2014). The solid-liquid interaction is 
incorporated via the adhesion work and the surface diffusion (Park and Aluru, 2010, 2007; Wei et al., 2011). They found that the effect of the physical adsorption on the oil flow is probably negligible. The critical pore radius when the boundary slip comes into play is provided. At the macroscale, researchers (Cui et al., 2018; Javadpour et al., 2015; Zhang et al., 2017) investigated the effects of the organic matter (Xi and Congli, 2015) on the liquid flow in shales in different views. However, at the pore scale, the impact of the prevalent mixed wettability (Habibi et al., 2016; Hu et al., 2014; Lu et al., 2012) has not been discussed. Moreover, light oil is assumed in aforementioned papers. Actually, the wettability alteration (Behbahani et al., 2014) and other mechanisms could result from the adsorption of asphaltenes in shale oil (Qin et al., 2000; Shedid et al., 2006). Nevertheless, there has been no research simultaneously quantifying the impacts of the mixed wettability and asphaltenes in shale oil rocks.

In this paper, the mathematical model incorporating the effects of the originally mixed wettability and the mechanisms triggered by asphaltenes is established. Comprehensive sensitivity analysis and comparisons with simplified models are carried out to demonstrate the crude oil flow mechanisms in shales. On the other hand, the effect of different adsorption patterns is briefly discussed.

\section{Mathematical model}

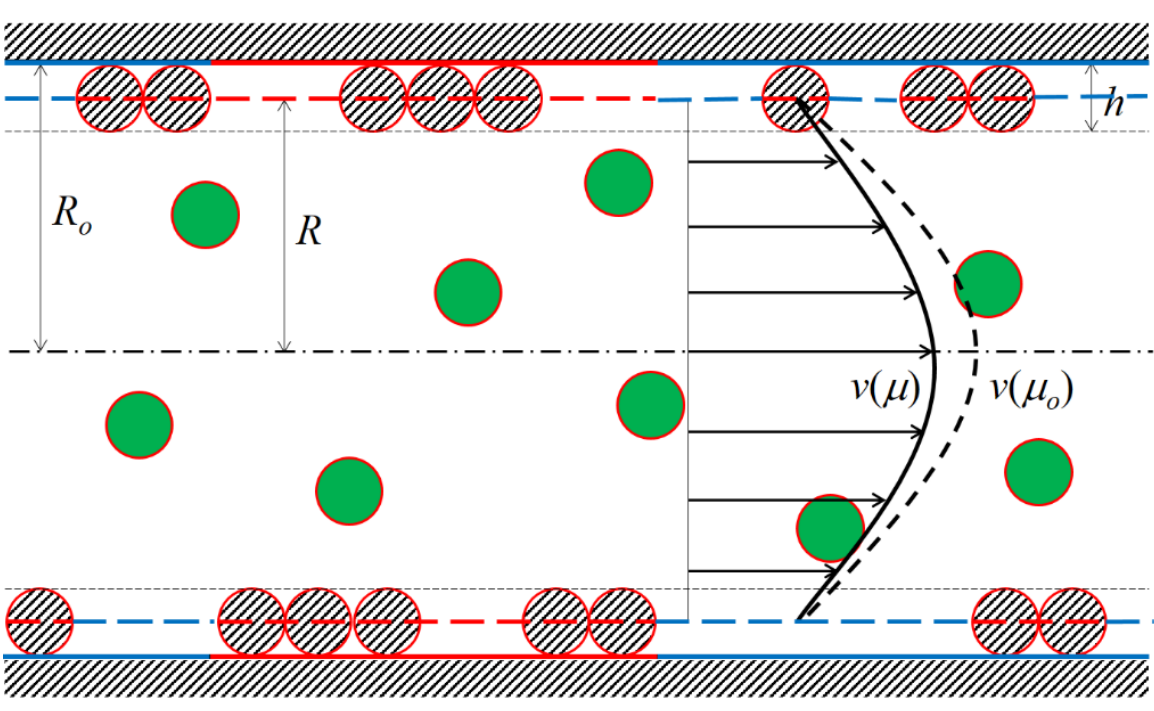

Symbol

Free particle Deposited particle -. - Center line Water-wet interface Oil-wet interface $\longrightarrow \begin{aligned} & \text { Flow } \\ & \text { velocity }\end{aligned}$ Equivalent interface 
In this section, a mathematical model for the apparent permeability of shale oil considering

70

71

72

light oil as well as asphaltenes in dual-wettability shales is established. As shown in Fig. 1 (the conceptual model), the two states of asphaltenes, free and deposited, are considered. The free asphaltenes increase the viscosity of shale oil, and the deposited asphaltenes can narrow the flow conduit and change the initial mixed wettability of the substrate. It should be noted that the particles can be asphaltene molecules, nanoaggregates or clusters. On the other hand, the particles can stack several layers (Behbahani et al., 2014; Betancourt et al., 2016; Jafari Behbahani et al., 2013), and only one layer is shown for convenience.

The mathematical model to be established is based on the flow enhancement $\lambda$ of a nanopore is (Mattia and Calabro, 2012):

$$
\lambda=1+\frac{8 \mu}{R^{2}} \frac{D_{s} L}{W_{A}}
$$

where $\mu$ is the viscosity, $R$ is the pore radius, $D_{s}$ is the surface diffusion, $W_{A}$ is the adhesion work, and $L$ is the pore length.

\subsection{Effect of the mixed wettability}

In this work, both organic and inorganic surfaces are considered. To incorporate the mixed wettability property, the equivalent expressions for the surface diffusion and the adhesion work should be obtained. The equivalent adhesion work can be achieved by the arithmetic average:

$$
W_{A}=(1-\alpha) W_{A, o}+\alpha W_{A, w}
$$

where $\alpha$ is the area ratio of the water-wet surface, subscripts $\mathrm{o}$ and $\mathrm{w}$ stand for oil-wet and water-wet surfaces, respectively. However, the treatment of the equivalent surface diffusion is different. With comparison to the 2D permeability upscaling formula (Naraghi and Javadpour, 2015): 


$$
k_{L B}=\frac{N_{c}}{N_{r}} \sum_{i=1}^{N_{r}} \frac{1}{\sum_{j=1}^{N_{c}} \frac{1}{k_{i, j}}}
$$

$$
k_{U B}=\frac{N_{c}}{N_{r}} \frac{1}{\sum_{j=1}^{N_{c}} \frac{1}{\sum_{i=1}^{N_{r}} k_{i, j}}}
$$

93

$$
k_{e q}=\sqrt{k_{U B} k_{L B}}
$$

where subscripts UB and LB stand for the upper bound and the lower bound respectively, $k_{e q}$ is its statistical average, $N_{c}$ is the number of grids in columns, $N_{r}$ is the number of grids in rows, $k_{i, j}$ is the permeability of the grid $(i, j)$. In case that both surfaces are distributed evenly, the equivalent surface diffusion can be calculated as follows:

$$
\begin{gathered}
D_{s, L B}=\frac{N_{c}}{N_{r}} \sum_{i=1}^{N_{r}} \frac{1}{\sum_{j=1}^{N_{c}} \frac{1}{D_{s, i, j}}}=\frac{1}{\frac{\alpha}{D_{s, w}}+\frac{1-\alpha}{D_{s, o}}} \\
D_{s, U B}=\frac{N_{c}}{N_{r}} \frac{1}{\sum_{j=1}^{N_{c}} \frac{1}{\sum_{i=1}^{N_{r}} D_{s, i, j}}}=\alpha D_{s, w}+(1-\alpha) D_{s, o} \\
D_{s}=\sqrt{D_{s, U B} D_{s, L B}}=\sqrt{\frac{\alpha D_{s, w}+(1-\alpha) D_{s, o}}{\frac{\alpha}{D_{s, w}}+\frac{1-\alpha}{D_{s, o}}}}
\end{gathered}
$$

101 where $D_{s}$ is its statistical average, and $D_{s, i, j}$ is the surface diffusion of the unit $(i, j)$. $\alpha$ imposes

102 complicated impacts on $D_{s}$, and $D_{s}$ should decrease with enlarging $\alpha$ (Appendix A provides a 103 detailed explanation).

\subsection{Effect of asphaltenes}

Asphaltenes can influence the hydrocarbon effective mobility mainly by the pore radius reduction, the wettability alteration, and the viscosity increment (Jafari Behbahani et al., 2013). In 
107

108

this section, their respective modeling methods are discussed. In view of the complexities of the equilibrium and realistic different structures (i.e., molecules, nanoaggregates or clusters) for asphaltenes, the ultimate adsorption thickness and the surface coverage are directly taken for discussions. Actually the referred adsorption also includes the deposition, but for concision, only the adsorption is referred in following discussions. It should be noted that the variation in the oil composition will lead to slightly different physical properties (i.e., the surface diffusion, the adhesion work, and the density), but this deviation is not incorporated in this work for simplicity.

\subsubsection{Wettability alteration}

In this preliminary investigation, for the pore surface covered by asphaltenes, it is assumed that its property is the same as the originally organic surface. Consequently, the initial water-wet and oil-wet surface ratios should be adjusted according to the surface coverage of adsorbed asphaltenes to determine the flow enhancement accurately. Assuming that adsorbed asphaltenes are randomly distributed (independent of the original wettability), the water-wet area ratio should be corrected as follows:

$$
\alpha=\alpha_{o} \cdot(1-\gamma)
$$

where $\gamma$ is the surface coverage of asphaltenes. Correspondingly, the oil-wet area ratio changes from $\left(1-\alpha_{o}\right)$ to $(1-\alpha)$.

\subsubsection{Pore radius reduction}

Due to the ultra-low mobility of adsorbed asphaltenes (Pan et al., 2005; Qin et al., 2000), the pore radius should be reduced to represent the resulting reduction in the flow capacity (a detailed derivation is provided in Appendix B):

$$
R=R_{o}-\gamma h
$$


129

130

131

132

134

135

136

137

138

139

140

141

142

143

144

145

146

147

148

149

where $h$ is the adsorption thickness. The flow enhancement with the corrected pore radius does not accord to the original pore radius. To accurately describe the flow deviation from the original case, the final expression of the flow enhancement should be multiplied by $\left(1 / 8 R^{2}\right) /\left(1 / 8 R_{o}^{2}\right)=\left(R / R_{o}\right)^{2}$.

\subsubsection{Viscosity increment}

Actually, the viscosity of movable oil depends on its containing fraction of asphaltenes, and the classical Einstein's equation is utilized to depict such relationship (Ghanavati et al., 2013):

$$
\mu=\mu_{o}(1+2.5 \varphi)
$$

where $\mu_{o}$ is the viscosity of light oil (contains no asphaltenes), and $\varphi$ is the volume fraction of asphaltenes in the free phase. However, it should be noted that the original concept of the flow enhancement is directly related to the "permeability", and does not have a direct relationship with the "viscosity". Therefore, the final flow enhancement should be divided by the viscosity increment factor $(1+2.5 \phi)$. The treatments with the viscosity here might seem redundant, but the constant "1" in Eq. (1) makes them plausible. The notation of the flow enhancement in this paper is similar to the "mobility", which is the ratio of the permeability to the viscosity. Actually, the viscosity change will lead to a significant change in the "permeability", but only a slight change in the "flow enhancement/mobility".

Generally, there is an exponential relationship between the oil viscosity and the asphaltene content. Nevertheless, it is well-known that oil is already difficult to move in the ultra-tight shale matrix, and thus shale oil with high asphaltene content would be extremely difficult to be exploited economically. Therefore, low asphaltenes content is assumed in this paper. In other words, the linear relationship between the oil viscosity and asphaltene content holds. 


\subsubsection{Volume conservation}

The pressure/temperature/time changes can lead to the dissolution or deposition of asphaltenes (Wang and Civan, 2005; Zendehboudi et al., 2013), but its total volume fraction $\beta$ should remain constant:

$$
\varphi \cdot \pi R^{2}+\pi\left(R_{o}^{2}-R^{2}\right)=\beta \cdot \pi R_{o}^{2}
$$

Eq. (12) can be transformed into:

$$
\varphi=1-(1-\beta)\left(\frac{R_{o}}{R}\right)^{2}
$$

\subsection{Mathematical model}

From all the above discussions, the full expression for the flow enhancement is reached:

$$
\begin{aligned}
& \left.\lambda=\left\{1+\frac{8 \mu_{o}\left[3.5-2.5(1-\beta)\left(\frac{R_{o}}{R_{o}-\gamma h}\right)^{2}\right]}{\left(R_{o}-\gamma h\right)^{2}}\right] \frac{\sqrt{\frac{\alpha_{o} \cdot(1-\gamma) D_{s, w}+\left[1-\alpha_{o} \cdot(1-\gamma)\right] D_{s, o}}{\frac{\alpha_{o} \cdot(1-\gamma)}{D_{s, w}}} L \frac{1-\alpha_{o} \cdot(1-\gamma)}{D_{s, o}}}}{\left[1-\alpha_{o} \cdot(1-\gamma)\right] W_{A, o}+\alpha_{o} \cdot(1-\gamma) W_{A, w}}\right\} \\
& \frac{\left(\frac{R_{o}-\gamma h}{R_{o}}\right)^{2}}{3.5-2.5(1-\beta)\left(\frac{R_{o}}{R_{o}-\gamma h}\right)^{2}}
\end{aligned}
$$

To make the form of Eq. (14) simpler, the concept of the relative adsorption thickness $h_{r}$ is introduced:

$$
h_{r}=\frac{h}{R_{o}}
$$

Therefore, Eq. (14) is simplified as: 


$$
\lambda=\left\{1+\frac{8 \mu_{o}\left[3.5-2.5(1-\beta)\left(\frac{1}{1-\gamma h_{r}}\right)^{2}\right]}{R_{o}^{2}\left(1-\gamma h_{r}\right)^{2}} \frac{\sqrt{\frac{\alpha_{o} \cdot(1-\gamma) D_{s, w}+\left[1-\alpha_{o} \cdot(1-\gamma)\right] D_{s, o}}{\frac{\alpha_{o} \cdot(1-\gamma)}{D_{s, w}}+\frac{1-\alpha_{o} \cdot(1-\gamma)}{D_{s, o}}}} L}{\left[1-\alpha_{o} \cdot(1-\gamma)\right] W_{A, o}+\alpha_{o} \cdot(1-\gamma) W_{A, w}}\right\}
$$$$
\frac{\left(1-\gamma h_{r}\right)^{2}}{3.5-2.5(1-\beta)\left(\frac{1}{1-\gamma h_{r}}\right)^{2}}
$$

\section{(16)}

It is apparent that the relationship between $\lambda$ and $R_{o}$ is the same as our previous work (Cui et al., 2017). This phenomena further strengthens the rationale of the introduction of $h_{r}$. Practically it is rather difficult to isolate some correction factors and research them in detail: the term $\left(1-\gamma h_{r}\right)^{2}$ accounts for the effect of the pore radius reduction, but it is contained in the expression of the viscosity increment; $(1-\gamma)$ appears many times in different places in the expressions for the equivalent surface diffusion and adhesion work, and therefore it is not convenient to isolate the impact of the wettability alteration.

To investigate the contributions of each stated mechanism, several simplified expressions of Eq. (16) are listed below for comparisons and analysis.

(1) $\gamma=0, h_{r}=0, \beta=0$ (only light oil):

$$
\lambda=1+\frac{8 \mu_{o}}{R_{o}^{2}} \frac{\sqrt{\frac{\alpha_{o} D_{s, w}+\left(1-\alpha_{o}\right) D_{s, o}}{\frac{\alpha_{o}}{D_{s, w}}+\frac{1-\alpha_{o}}{D_{s, o}}}} L}{\left(1-\alpha_{o}\right) W_{A, o}+\alpha_{o} W_{A, w}}
$$


180

$$
\lambda=\left\{1+\frac{8 \mu_{o}\left[3.5-2.5(1-\beta)\left(\frac{1}{1-\gamma h_{r}}\right)^{2}\right]}{R_{o}^{2}\left(1-\gamma h_{r}\right)^{2}} \frac{\sqrt{\frac{\alpha_{o} D_{s, w}+\left(1-\alpha_{o}\right) D_{s, o}}{\frac{\alpha_{o}}{D_{s, w}}+\frac{1-\alpha_{o}}{D_{s, o}}}} L}{\left(1-\alpha_{o}\right) W_{A, o}+\alpha_{o} W_{A, w}}\right\} \cdot \frac{\left(1-\gamma h_{r}\right)^{2}}{3.5-2.5(1-\beta)\left(\frac{1}{1-\gamma h_{r}}\right)^{2}}
$$

181

(3) Ignore the pore radius reduction expressed in Eq. (10):

$$
\lambda=\left\{1+\frac{8 \mu_{o}\left[3.5-2.5(1-\beta)\left(\frac{1}{1-\gamma h_{r}}\right)^{2}\right]}{R_{o}^{2}} \frac{\sqrt{\frac{\alpha_{o} \cdot(1-\gamma) D_{s, w}+\left[1-\alpha_{o} \cdot(1-\gamma)\right] D_{s, o}}{\frac{\alpha_{o} \cdot(1-\gamma)}{D_{s, w}}+\frac{1-\alpha_{o} \cdot(1-\gamma)}{D_{s, o}}}} L}{\left[1-\alpha_{o} \cdot(1-\gamma)\right] W_{A, o}+\alpha_{o} \cdot(1-\gamma) W_{A, w}}\right\}
$$

$$
\frac{1}{3.5-2.5(1-\beta)\left(\frac{1}{1-\gamma h_{r}}\right)^{2}}
$$

(4) Ignore the viscosity increment expressed in Eq. (11):

$$
\lambda=\left\{1+\frac{8 \mu_{o}}{R_{o}^{2}\left(1-\gamma h_{r}\right)^{2}} \frac{\sqrt{\frac{\alpha_{o} \cdot(1-\gamma) D_{s, w}+\left[1-\alpha_{o} \cdot(1-\gamma)\right] D_{s, o}}{\frac{\alpha_{o} \cdot(1-\gamma)}{D_{s, w}}+\frac{1-\alpha_{o} \cdot(1-\gamma)}{D_{s, o}}}}}{\left[1-\alpha_{o} \cdot(1-\gamma)\right] W_{A, o}+\alpha_{o} \cdot(1-\gamma) W_{A, w}}\right\} \cdot\left(1-\gamma h_{r}\right)^{2}
$$

(5) Ignore the slip enhancement (micro-fractures, $R_{o}$ is large enough):

$$
\lambda=\frac{\left(1-\gamma h_{r}\right)^{2}}{3.5-2.5(1-\beta)\left(\frac{1}{1-\gamma h_{r}}\right)^{2}}
$$

For the common scale of micro-fracture apertures, the slip enhancement is negligible (as 
191 fracture surfaces are usually planar. Nevertheless, the regularities should be similar, and the results

192 should systematically deviate to some extent. The accurate modeling of shale micro-fractures will

193 be discussed in our forthcoming article.

194 3. Results and discussions

195 Table 1

196 Parameters for analysis

\begin{tabular}{cccccc}
\hline Parameter & $L$ & $\mu_{o}$ & $\beta$ & $h_{r}$ & \\
\hline Value & $2 \mu \mathrm{m}$ & $0.8 \mathrm{mPa} \cdot \mathrm{s}$ & 0.5 & 0.2 & \\
\hline Parameter & $\gamma$ & $D_{s, w}$ & $D_{s, o}$ & $W_{A, o}$ & $W_{A, w}$ \\
\hline Value & 0.5 & $0.3 \times 10^{-9} \mathrm{~m}^{2} / \mathrm{s}$ & $3 \times 10^{-9} \mathrm{~m}^{2} / \mathrm{s}$ & $0.1 \mathrm{~J} / \mathrm{m}^{2}$ & $1 \mathrm{~J} / \mathrm{m}^{2}$ \\
\hline
\end{tabular}

197

The basic parameters for sensitivity analysis are set as Table 1. There are significant differences in the size and the wettability between inorganic and organic pores, and these differences probably lead to distinct flow characteristics. Thus, the two pore types are discussed respectively in following discussions. Organic pores dominate in several nanometers and tend to be oil-wet, and $R_{o}=5 \mathrm{~nm}, \alpha_{o}=0.2$ are set. The radius of mainly water-wet inorganic pores mostly lies in tens of nanometers, and $R_{o}=20 \mathrm{~nm}$ and $\alpha_{o}=0.8$ are set for them. Besides, micro-fractures are prevalent in shale formations, thus $R_{o}=1000 \mathrm{~nm}$ and $\alpha_{o}=0.5$ are taken for them. Actually, the aperture of micro-fractures mostly lies between $1 \mu \mathrm{m}$ and $100 \mu \mathrm{m}$. However, the increase of aperture imposes a negligible impact on the respective contributions from each mechanism if the aperture is beyond $1 \mu \mathrm{m}$ (as illustrated in Fig. 2). On the other hand, the effect of the wettability is negligible at this scale (Cui et al., 2017), and the actual value of $\alpha_{o}$ will not affect the results.

From Eq. (16), there are altogether 11 variables: $\mu_{o}, \beta, R_{o}, \gamma, h_{r}, \alpha_{o}, D_{s, w}, D_{s, o}, W_{A, o}, W_{A, w}, L$. 
209

210

Among them, $D_{s, w}, D_{s, o}, W_{A, o}, W_{A, w}, L$, and $\mu_{o}$ are of little interest to this work, and can be regarded

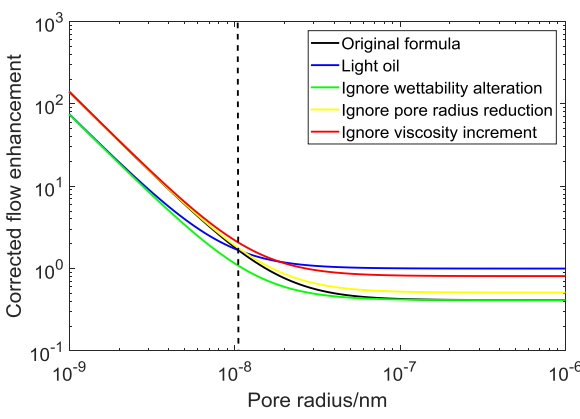

(a)

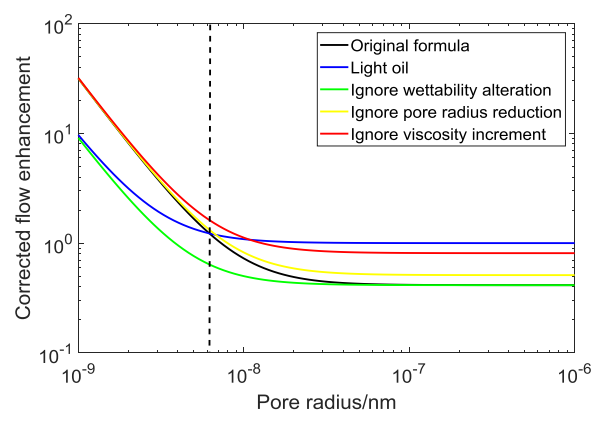

(b)

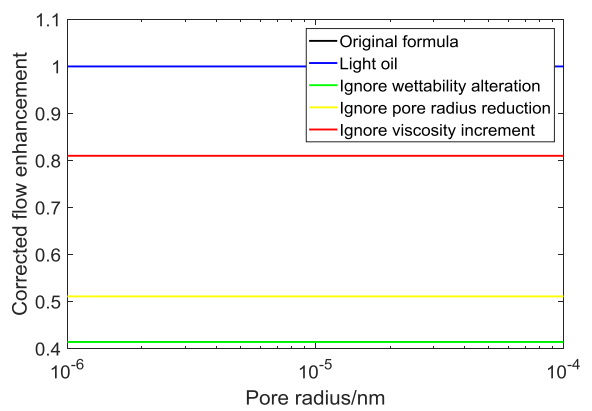

(c) 
micro-fractures, the interval is $[1 \mu \mathrm{m}, 100 \mu \mathrm{m}]$. (For interpretation of the references to color in this figure legend,

the reader is referred to the web version of this article.)

From Fig. 2, the flow enhancement of organic pores is very sensitive (decreases rapidly) to

the pore radius (mainly several nanometers), and for inorganic pores (mainly tens of nanometers)

it is much less sensitive, while for micro-fractures the aperture has no contribution to the flow enhancement.

It is evident that the relative importance of all considered mechanisms will change with the increment of the pore radius for organic and inorganic pores. While for micro-fractures, there is no such change. At the relatively small pore scale, the wettability alteration contributes more than others. As the pore radius increases, the viscosity increment and the pore radius reduction gradually come into stage and contribute more than the wettability alteration. For large enough pores (and micro-fractures), the influence of the wettability alteration is negligible. Therefore, the wettability alteration is only meaningful to the nanoscale oil flow, while the viscosity increment and the pore radius reduction should be concerned for only macroscale applications. This is the fundamental difference among them.

In another view, the addition of asphaltenes will enhance the permeability if the pore radius is below about $10 \mathrm{~nm}$, and impair the flow conductance when the pore radius is beyond about $10 \mathrm{~nm}$. This critical value $(10 \mathrm{~nm})$ is slightly larger for organic pores, and for inorganic pores it is slightly smaller, while there is no such critical value for micro-fractures. 


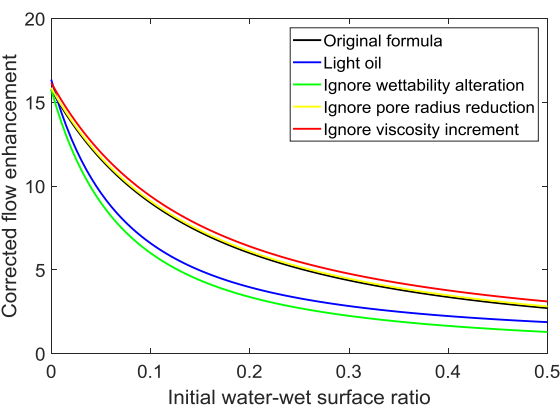

(a)

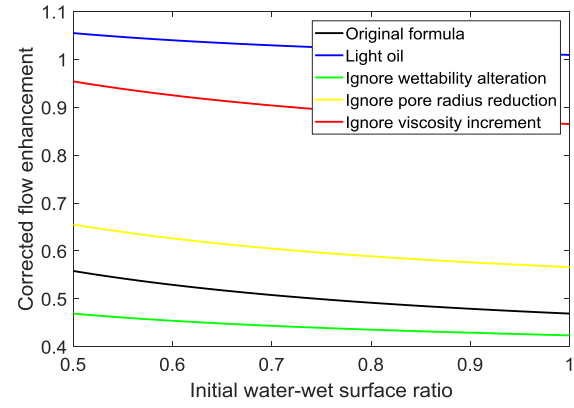

(b)

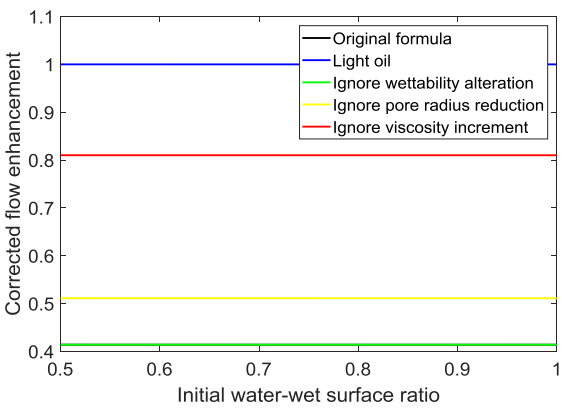

(c)

Fig. 3. Effect of the initial water-wet surface ratio. (a): analysis for organic pores. (b): analysis for inorganic pores.

(c): analysis for micro-fractures. It should be noted that $\alpha_{o}$ is set as intervals instead of specific values here, as $\alpha_{o}$

is right the variable for sensitivity analysis. (For interpretation of the references to color in this figure legend, the reader is referred to the web version of this article.)

As shown in Fig. 3, the oil flow in shale organic nanopores is very sensitive to the initial wettability condition of the substrate: with the increase of the initial water-wet surface ratio, the

flow enhancement decreases rapidly. For inorganic nanopores, the relationship is much less sensitive. As for micro-fractures, there is no such sensitivity.

Apparently, for organic pores, the contribution of the wettability alteration is primary unless the initial water-wet surface ratio is extremely small. In essence, more water-wet surface ratio offers more chances for the wettability alteration to come into play. Comparatively speaking, the 
260

261

262

264

265

266

rank for inorganic pores and micro-fractures is totally different. The viscosity increment and the (c): analysis for micro-fractures. It should be noted that the interval for sensitivity analysis here is not $[0,1]$,

pore radius reduction have greater contributions compared to organic pores.

In another view, the addition of asphaltenes will enhance the permeability of organic pores, while reduce the permeability of inorganic pores and micro-fractures. The contribution of asphaltenes is nearly insensitive to the initial wettability condition (the initial water-wet surface ratio) for inorganic pores. For micro-fractures, such contribution is totally insensitive. While for organic pores, moderate initial water-wet surface ratio is most beneficial for its permeability enhancement.

\subsection{Effect of relative adsorption thickness $\left(h_{r}\right)$}

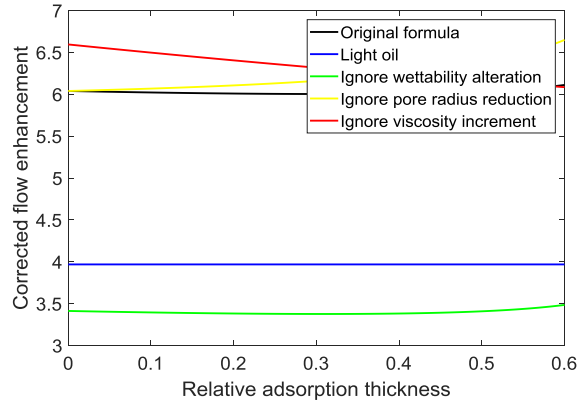

(a)

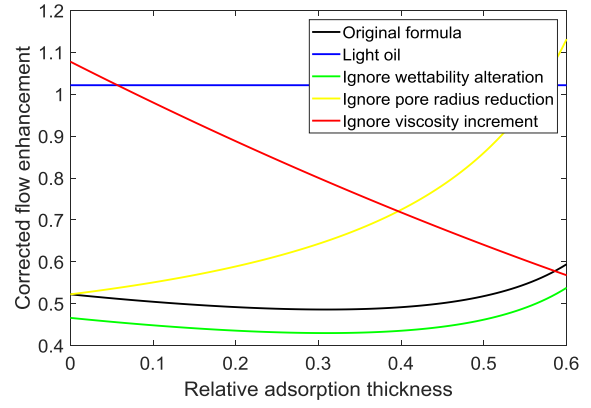

(b)

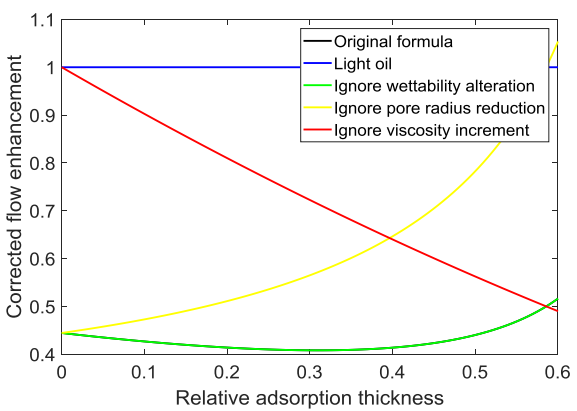

(c)

Fig. 4. Effect of the relative adsorption thickness. (a): analysis for organic pores. (b): analysis for inorganic pores.

because the relative adsorption thickness is subject to the total volume fraction $\beta$ (Eq. (12)). [0, 0.6] is the 
maximum rational range after some calculations. (For interpretation of the references to color in this figure legend,

the reader is referred to the web version of this article.)

As illustrated in Fig. 4, the flow enhancement values of organic and inorganic pores, as well

as micro-fractures, are all not sensitive to the adsorption thickness: the change of the permeability of organic pores is not easily observable, while inorganic pores and micro-fractures experience a slight decrease and then a slight increase in the permeability with enlarging adsorption thickness.

\subsection{Effect of surface coverage $(\gamma)$}
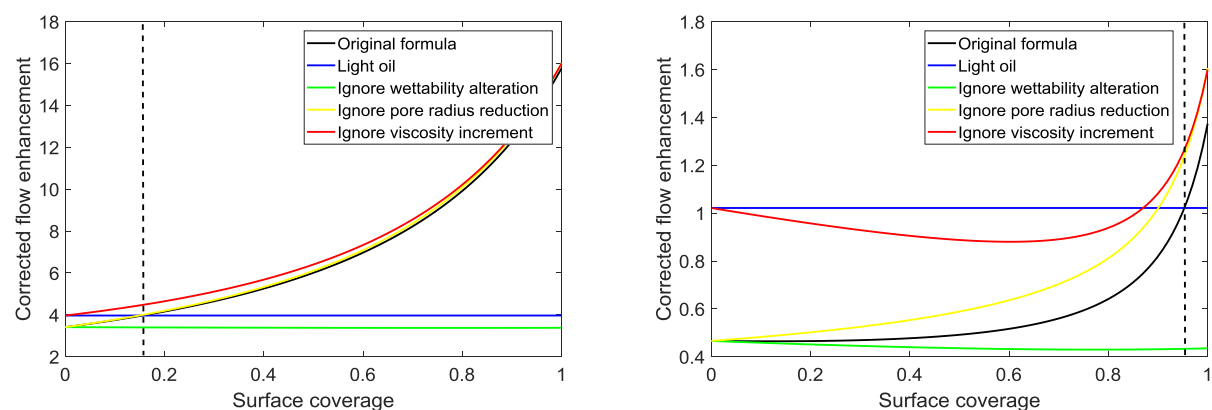
296

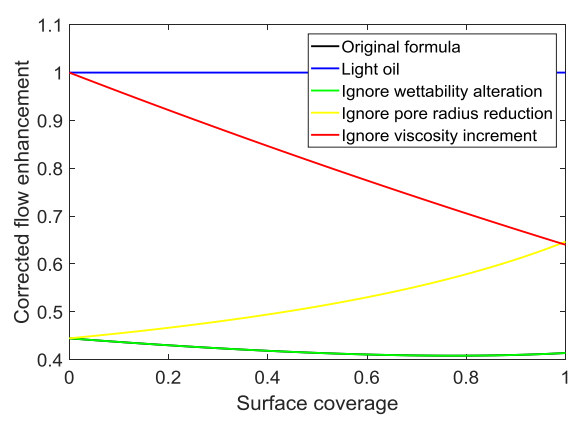

(c)

Fig. 5. Effect of the surface coverage. (a): analysis for organic pores. (b): analysis for inorganic pores. (c): analysis for micro-fractures. (For interpretation of the references to color in this figure legend, the reader is referred to the web version of this article.)

Fig. 5 shows the positive contribution of the surface coverage to the flow enhancement for organic pores, while for inorganic pores this enhancement is only observable at a high surface coverage. Interestingly, the effect on micro-fractures can be slightly negative.

Increasing surface coverage will also lead to the re-rank of the mechanisms. For organic pores, the effect of the pore radius reduction is negligible throughout. Only the viscosity increment plays a role when the surface coverage is relatively low, while the wettability alteration is dominant if the surface coverage is larger. The explanation for this subsection is the most complicated, because all proposed mechanisms are of concern. Firstly, on condition that other variables are not changed, more coverage of asphaltenes implies less free asphaltenes, resulting in a lower oil viscosity and a greater flow speed. Secondly, a larger coverage will result in more oil-wet surface (the wettability alteration), and this contributes to a greater transport velocity. Thirdly, the organic permeability is insensitive to the pore radius (Cui et al., 2017), and therefore the contribution of the pore radius reduction is negligible. Contrary to organic pores, the impact of the pore radius 
314

315

318

319

320

321

322

323

324

325

326

327

328

329

330

331

332

333

334

335

reduction is observable for inorganic pores and micro-fractures, and the viscosity increment also plays a more important role, while the influence from the wettability alteration is far less important.

In another view, the addition of asphaltenes will enhance the permeability of organic pores unless the surface coverage is small enough, while reduce the permeability of inorganic pores unless the surface coverage is large enough. While for micro-fractures, this contribution is totally negative. Obviously, the contribution of asphaltenes is intimately related to the surface coverage for organic and inorganic pores. As for micro-fractures, there is no such relevance.

\subsection{Effect of adsorption pattern $\left(h_{r}\right.$ and $\left.\gamma\right)$}

In this paper, the adsorption pattern (or the solution of asphaltenes) is characterized and can be fully determined by the relative adsorption thickness and the surface coverage. Based on the results from Sec. 3.3 and Sec 3.4, it can be concluded that for organic and inorganic pores the flow enhancement can vary significantly with different adsorption patterns if the adsorbed amount (or the dissolved amount) keeps constant. However, for micro-fractures, such variance is not obvious. In other words, accurately depicting the adsorption pattern, not just the adsorbed amount (or the dissolved amount), is essential for precisely determining the flow capacity of shale nanopores (except micro-fractures). To be more exact, a flat configuration of adsorbed asphaltenes is favorable for the oil flow capacity of shale nanopores with a fixed amount of adsorbed asphaltenes.

Nevertheless, for micro-fractures, there is no such regularity.

\section{Conclusions}

This work established a mathematical model for the shale oil permeability including the effects of the mixed wettability and asphaltenes. As for asphaltenes, the effects of the wettability 
336 alteration, the pore radius reduction, and the viscosity increment are incorporated. The main findings are summarized as follows:

(1) The sensitivity to each variable. The flow enhancement is not sensitive to the adsorption thickness. As for the initial water-wet surface ratio, the surface coverage, and the pore radius, the flow enhancement of organic pores is more sensitive than inorganic nanopores, and then micro-fractures. The contribution of the surface coverage is positive, while that of the rest two factors is negative.

(2) The essence of mechanisms. The wettability alteration is only meaningful to the nanoscale oil flow (mainly organic pores), and relevant to the initial water-wet surface ratio and the surface coverage. While the viscosity increment and the pore radius reduction should be concerned for only macroscale applications (mainly inorganic pores and micro-fractures), and they are both relevant to the relative adsorption thickness and the surface coverage.

(3) Effect of asphaltenes. Generally, the addition of asphaltenes will enhance the permeability of organic pores, while reduce the permeability of inorganic pores and micro-fractures. The contribution of asphaltenes is not related to the adsorption thickness, but relevant to the surface coverage and the pore radius (except micro-fractures). As for the initial water-wet surface ratio, it differs between organic pores and inorganic pores \& micro-fractures.

(4) Effect of the adsorption pattern. A flat configuration of adsorbed asphaltenes is relatively favorable for the oil flow capacity of shale nanopores, while for micro-fractures the configuration makes a negligible contribution.

\section{Nomenclature}

MDS Molecular dynamics simulation 


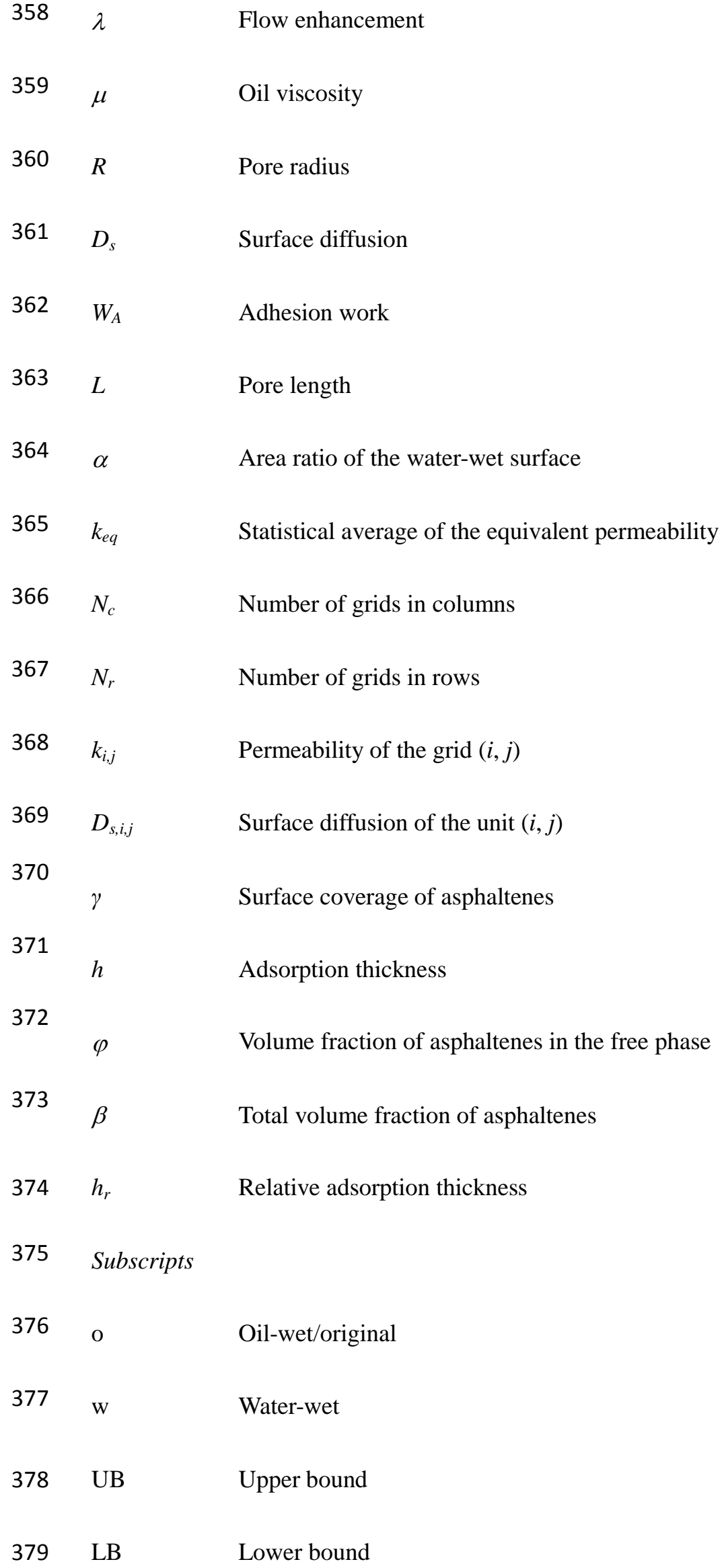




\section{References}

Behbahani, T.J., Ghotbi, C., Taghikhani, V., Shahrabadi, A., 2014. Investigation of asphaltene adsorption in sandstone core sample during $\mathrm{CO} 2$ injection: Experimental and modified modeling. Fuel 133, 63-72. https://doi.org/10.1016/j.fuel.2014.04.079

Betancourt, S.S., Ventura, G.T., Pomerantz, A.E., Viloria, O., Dubost, F.X., Zuo, J., Monson, G., Bustamante, D., Purcell, J.M., Nelson, R.K., Rodgers, R.P., Reddy, C.M., Marshall, A.G., Mullins, O.C., 2016. Nanoaggregates of Asphaltenes in a Reservoir Crude Oil and Reservoir Connectivity $\dagger$. Energy and Fuels 1178-1188.

Botan, A., Rotenberg, B., Marry, V., Turq, P., Noetinger, B., 2011. Hydrodynamics in clay nanopores. J. Phys. Chem. C 115, 16109-16115. https://doi.org/10.1021/jp204772c

Bousige, C., Ghimbeu, C.M., Vix-Guterl, C., Pomerantz, A.E., Suleimenova, A., Vaughan, G., Garbarino, G., Feygenson, M., Wildgruber, C., Ulm, F.-J., Pellenq, R.J.-M., Coasne, B., 2016. Realistic molecular model of kerogen's nanostructure. Nat. Mater. 15, 576-82. https://doi.org/10.1038/nmat4541

Cui, J., Cheng, L., Li, L., 2018. Apparent permeability and representative size of shale: a numerical study on the effects of organic matter. Comput. Geosci. https://doi.org/10.1007/s10596-018-9739-3

Cui, J., Sang, Q., Li, Y., Yin, C., Li, Y., Dong, M., 2017. Liquid permeability of organic nanopores in shale: Calculation and analysis. Fuel 202, 426-434. https://doi.org/10.1016/j.fuel.2017.04.057

Falk, K., Coasne, B., Pellenq, R., Ulm, F., Bocquet, L., 2015. Subcontinuum mass transport of condensed hydrocarbons in nanoporous media. Nat. Commun. 1-7. https://doi.org/10.1038/ncomms7949 
402 Ghanavati, M., Shojaei, M.J., Ahmad Ramazani, S.A., 2013. Effects of asphaltene content and 403 temperature on viscosity of Iranian heavy crude oil: Experimental and modeling study. Energy and Fuels 27, 7217-7232. https://doi.org/10.1021/ef400776h

Habibi, A., Dehghanpour, H., Binazadeh, M., Bryan, D., Uswak, G., 2016. Advances in Understanding Wettability of Tight Oil Formations: A Montney Case Study. SPE Reserv. Eval. Eng. 19, 583-603. https://doi.org/10.2118/175157-PA

Ho, A.T., Papavassiliou, D. V, Lee, L.L., Striolo, A., 2011. Liquid water can slip on a hydrophilic $\begin{array}{lllll}\text { surface. } & \text { Proc. } & \text { Natl. } & \text { Acad. } & \text { Sci. }\end{array}$ https://doi.org/10.1073/pnas.1105189108/-/DCSupplemental.www.pnas.org/cgi/doi/10.1073/pnas .1105189108

Hu, Y., Devegowda, D., Striolo, A., Phan, A.T. Van, Ho, T.A., Civan, F., Sigal, R., 2014. Microscopic dynamics of water and hydrocarbon in shale-kerogen pores of potentially mixed wettability. SPE J. $20,112-124$.

Jafari Behbahani, T., Ghotbi, C., Taghikhani, V., Shahrabadi, A., 2013. Asphaltene deposition under dynamic conditions in porous media: Theoretical and experimental investigation. Energy and Fuels 27, 622-639. https://doi.org/10.1021/ef3017255

Javadpour, F., Mcclure, M., Naraghi, M.E., 2015. Slip-corrected liquid permeability and its effect on hydraulic fracturing and fluid loss in shale. Fuel 160, 549-559. https://doi.org/10.1016/j.fuel.2015.08.017

Li, Y., Xu, J., Li, D., 2010. Molecular dynamics simulation of nanoscale liquid flows. Microfluid. Nanofluidics 9, 1011-1031. https://doi.org/10.1007/s10404-010-0612-5

Lu, S., Huang, W., Chen, F., Li, J., Wang, M., Xue, H., Wang, W., Cai, X., 2012. Classification and 
evaluation criteria of shale oil and gas resources : Discussion and application. Pet. Explor. Dev. 39, 268-276. https://doi.org/10.1016/S1876-3804(12)60042-1

Mattia, D., Calabro, F., 2012. Explaining high flow rate of water in carbon nanotubes via solid-liquid molecular interactions. $\quad$ Microfluid. $\quad$ Nanofluidics $\quad 13, \quad 125-130$. https://doi.org/10.1007/s10404-012-0949-z

Mattia, D., Leese, H., Lee, K.P., 2015. Carbon nanotube membranes: From flow enhancement to permeability. J. Memb. Sci. 475, 266-272. https://doi.org/10.1016/j.memsci.2014.10.035

Naraghi, M.E., Javadpour, F., 2015. A stochastic permeability model for the shale-gas systems. Int. J. Coal Geol. 140, 111-124. https://doi.org/10.1016/j.coal.2015.02.004

Neto, C., Evans, D.R., Bonaccurso, E., Butt, H.-J., Craig, V.S.J., 2005. Boundary slip in Newtonian liquids: a review of experimental studies. Reports Prog. Phys. 68, 2859-2897. https://doi.org/10.1088/0034-4885/68/12/R05

Pan, C., Feng, J., Tian, Y., Yu, L., Luo, X., Sheng, G., Fu, J., 2005. Interaction of oil components and clay minerals in reservoir sandstones. Org. Geochem. 36, 633-654. https://doi.org/10.1016/j.orggeochem.2004.10.013

Park, J.H., Aluru, N.R., 2010. Ordering-induced fast diffusion of nanoscale water film on graphene. J. Phys. Chem. C 114, 2595-2599. https://doi.org/10.1021/jp907512z

Park, J.H., Aluru, N.R., 2007. Surface diffusion of n-alkanes: Mechanism and anomalous behavior. Chem. Phys. Lett. 447, 310-315. https://doi.org/10.1016/j.cplett.2007.09.047

Qin, X., Wang, P., Sepehrnoori, K., Pope, G.A., 2000. Modeling asphaltene precipitation in reservoir simulation. Ind. Eng. Chem. Res. 39, 2644-2654.

Ritos, K., Mattia, D., Calabrò, F., Reese, J.M., 2014. Flow enhancement in nanotubes of different 

materials and lengths. J. Chem. Phys. 140, 014702. https://doi.org/10.1063/1.4846300

Secchi, E., Marbach, S., Niguès, A., Stein, D., Siria, A., Bocquet, L., 2016. Massive radius-dependent flow slippage in carbon nanotubes. Nature 537, 210-213. https://doi.org/10.1038/nature19315

Shedid, S.A., U, S.C., Zekri, A.Y., U, U.A.E., 2006. Formation Damage Caused by Simultaneous Sulfur and Asphaltene Deposition. spe Prod. Oper. 18-20.

Strogatz, S.H., Abrams, D.M., McRobie, A., Eckhardt, B., Ott, E., 2005. Enhanced flow in carbon nanotubes. Nature 438, 43-44. https://doi.org/10.1038/43843a

Thomas, J.A., McGaughey, A.J.H., 2009. Water flow in carbon nanotubes: Transition to subcontinuum transport. Phys. Rev. Lett. 102, 184502. https://doi.org/10.1103/PhysRevLett.102.184502

Wang, H., Liu, Y., Li, M., Huang, H., Xu, H.M., Hong, R.J., Shen, H., 2010. Water flow enhancement in hydrophilic nanochannels. Nanoscale 4, 1166-1169. https://doi.org/10.1039/b000000x

Wang, S., Civan, F., 2005. Modeling Formation Damage by Asphaltene Deposition During Primary Oil Recovery. J. Energy Resour. Technol. 127, 310. https://doi.org/10.1115/1.1924465

Wang, S., Feng, Q., Javadpour, F., Xia, T., Li, Z., 2015a. Oil adsorption in shale nanopores and its effect on recoverable oil-in-place. Int. J. Coal Geol. 147-148, 9-24. https://doi.org/10.1016/j.coal.2015.06.002

Wang, S., Feng, Q., Zha, M., Lu, S., Qin, Y., Xia, T., Zhang, C., 2015b. Molecular dynamics simulation of liquid alkane occurrence state in pores and slits of shale organic matter. Pet. Explor. Dev. 42, 844-851. https://doi.org/10.11698/PED.2015.06.10

Wang, S., Javadpour, F., Feng, Q., 2016a. Molecular dynamics simulations of oil transport through inorganic nanopores in shale. Fuel 171, 74-86. https://doi.org/10.1016/j.fuel.2015.12.071

Wang, S., Javadpour, F., Feng, Q., 2016b. Fast mass transport of oil and supercritical carbon dioxide 
470

471

472

473

Wei, M.J., Zhou, J., Lu, X., Zhu, Y., Liu, W., Lu, L., Zhang, L., 2011. Diffusion of water molecules confined in slits of rutile TiO2(110) and graphite(0001). Fluid Phase Equilib. 302, 316-320. https://doi.org/10.1016/j.fluid.2010.09.044

Xi, Z., Congli, W., 2015. Analysis of Formation Conditions of Shale Oils in the Changling Depression , Sourthern Songliao Basin 2 Forming Conditions of Shale Oil. Acta Geol. Sin. 89, 332-334.

Zendehboudi, S., Ahmadi, M.A., Mohammadzadeh, O., Bahadori, A., Chatzis, I., 2013. Thermodynamic investigation of asphaltene precipitation during primary oil production: Laboratory and smart technique. Ind. Eng. Chem. Res. 52, 6009-6031. https://doi.org/10.1021/ie301949c

Zhang, Q., Su, Y., Wang, W., Lu, M., Sheng, G., 2017. Apparent permeability for liquid transport in nanopores of shale reservoirs: Coupling flow enhancement and near wall flow. Int. J. Heat Mass Transf. 115, 224-234. https://doi.org/10.1016/j.ijheatmasstransfer.2017.08.024

\section{Appendix A. Partial derivative for surface diffusion $\left(D_{s}\right)$}

The expression for the equivalent surface diffusion is simplified by taking its square:

$$
D_{s}^{2}=\frac{\alpha D_{s, w}+(1-\alpha) D_{s, o}}{\frac{\alpha}{D_{s, w}}+\frac{1-\alpha}{D_{s, o}}}
$$




$$
\begin{aligned}
& \frac{\partial D_{s}^{2}}{\partial \alpha}=\partial\left[\frac{\alpha D_{s, w}+(1-\alpha) D_{s, o}}{\left.\frac{\alpha}{D_{s, w}}+\frac{1-\alpha}{D_{s, o}}\right] / \partial \alpha}\right. \\
& =\frac{\left(D_{s, w}-D_{s, o}\right)\left(\frac{\alpha}{D_{s, w}}+\frac{1-\alpha}{D_{s, o}}\right)-\left[\alpha D_{s, w}+(1-\alpha) D_{s, o}\right]\left(\frac{1}{D_{s, w}}-\frac{1}{D_{s, o}}\right)}{\left(\frac{\alpha}{D_{s, w}}+\frac{1-\alpha}{D_{s, o}}\right)^{2}} \\
& =\frac{D_{s, w}-D_{s, o}}{D_{s, w} D_{s, o}} \frac{D_{s, o}+D_{s, w}}{\left(\frac{\alpha}{D_{s, w}}+\frac{1-\alpha}{D_{s, o}}\right)^{2}}<0
\end{aligned}
$$

Namely, as the water-wet area becomes more significant, the equivalent surface diffusion is

reduced. This regularity accords with the common sense.

\section{Appendix B. Derivation for equivalent radius}

The exact solution for the equivalent radius is derived at first, and then the rationale of the approximation is explained. The cross-section area belonging to asphaltenes $S$ is:

$$
S=\pi\left[R_{o}^{2}-\left(R_{o}-h\right)^{2}\right] \gamma
$$

The equivalent radius is deduced from the cross-section area for movable oil:

$$
\pi R^{2}+S=\pi R_{o}^{2}
$$

Based on Eqs. (23) and (24), the ultimate solution for $R$ is:

$$
\begin{aligned}
& R=\sqrt{R_{o}^{2}-\left(2 R_{o} h-h^{2}\right) \gamma}=R_{o} \sqrt{1-\left(2 h_{r}-h_{r}^{2}\right) \gamma} \\
& \approx R_{o} \sqrt{1-2 \gamma h_{r}} \approx R_{o}\left(1-\frac{2 \gamma h_{r}}{2}\right)=R_{o}-h \gamma
\end{aligned}
$$

Actually, the amount of asphaltenes is not significant in shale oil ( $h_{r}$ is negligible), thus the above approximation holds. 


\section{*Highlights}

(1) The effects of mixed wettability and asphaltenes on shale permeability are studied.

(2) Wettability alteration, pore radius reduction, and viscosity increase are considered.

(3) The differences among organic pores, inorganic pores and micro-fractures are shown.

(4) The respective contributions of all mechanisms to flow enhancement are investigated.

(5) Adsorption detail is important for flow in shale nanopores, not for micro-fractures. 


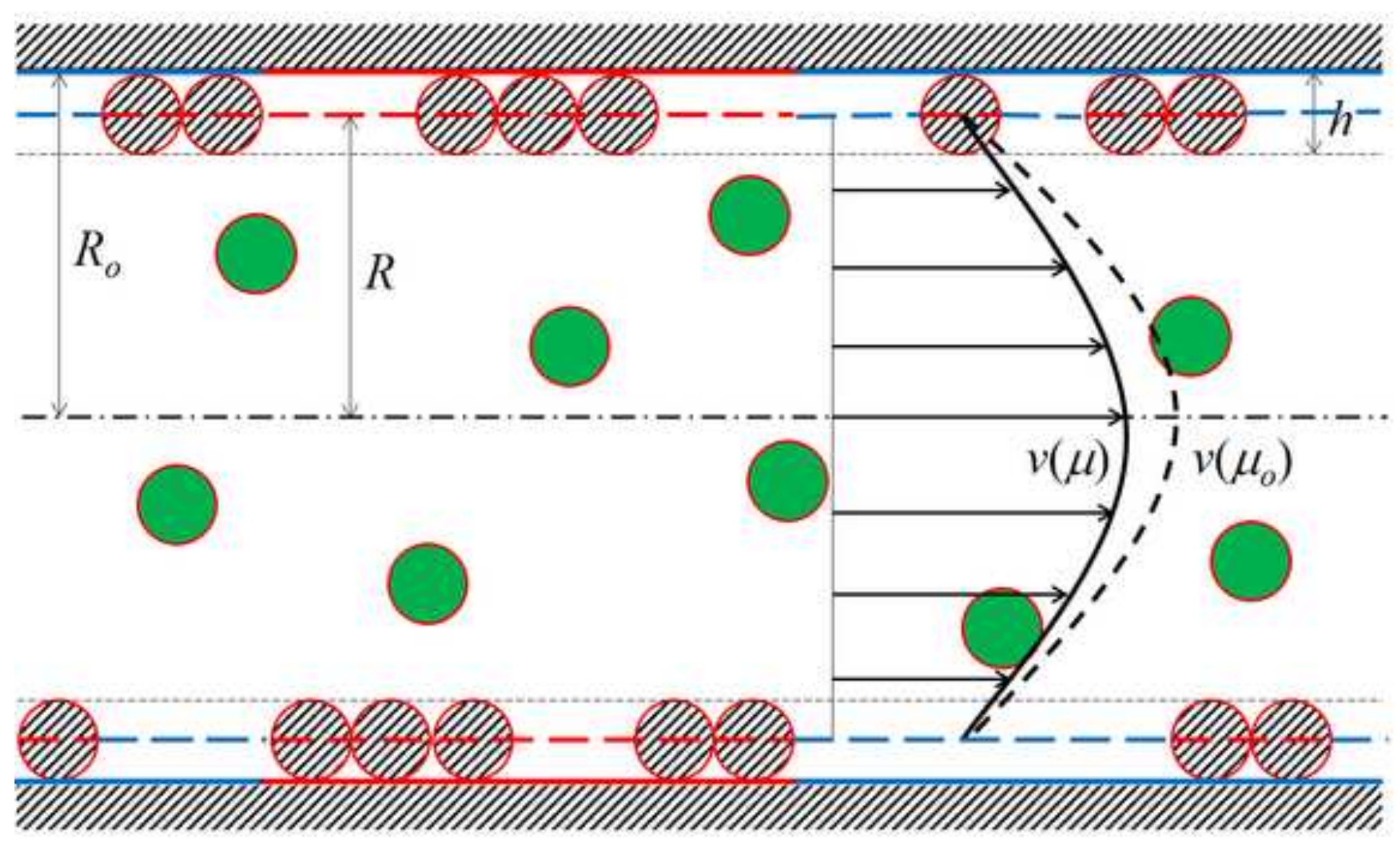

Symbol

Free particle

Deposited particle

-.- Center

line

- Water-wet interface

- Oil-wet interface

$\longrightarrow \begin{aligned} & \text { Flow } \\ & \text { velocity }\end{aligned}$ - - $\begin{aligned} & \text { Equivalent } \\ & \text { interface }\end{aligned}$ 